\title{
Design and Evaluation of Coexistence Mechanisms for Bluetooth and IEEE 802.11b Systems
}

\author{
Yu-Kwong Kwok and Michael Cho-Hoi Chek \\ Department of Electrical and Electronic Engineering \\ The University of Hong Kong, Pokfulam Road, Hong Kong \\ Corresponding Author: Yu-Kwong Kwok (Email: ykwok@hku.hk)
}

\begin{abstract}
Short-range wireless technologies are becoming increasingly important in enabling useful mobile applications. Bluetooth and IEEE 802.11b standards are the most commonly deployed technologies for WPAN and WLAN. However, because both standards share the same unlicensed ISM (Industrial, Scientific, Medical) radio spectrum, severe interference is inevitable and performance can be impaired significantly when heterogeneous devices using the two technologies come into close proximity. The most notable solution to this problem is a frequency domain non-collaborative coexistence mechanism called adaptive frequency hopping (AFH). However, we find that the efficiency of the "channel classification" sub-process in non-collaborative mechanisms is by and large ignored in the literature. Moreover, we also find that there is no system resources awareness and no interference source genre concerns in IEEE 802.15 Task Group 2 AFH (TG2 AFH) design. Thus, we suggest a new approach called ISOAFH (Interference Source Oriented AFH). With the above considerations, we propose a customized channel classification process, thereby simplifying the time and space complexity of the mechanism. Through our detailed implementation of various coexistence mechanisms in MATLAB Simulink, it is observed that TG2 AFH performance is sensitive to memory and power limitations, while ISOAFH is much less sensitive to these constraints and can keep a much lower channel collision rate. On the other hand, in this paper we also study some open issues of a time domain mechanism called MDMS (Master Delay MAC Scheduling). We compare different coexistence mechanisms and find that the performance of each approach very much depends on the efficiency of its sub-processes.

Keywords: mobile computing, adaptive frequency hopping, scheduling, coexistence, Bluetooth, IEEE $802.11 \mathrm{~b}$, IEEE $\mathbf{8 0 2 . 1 5}$, resource constraints.
\end{abstract}

\section{INTRODUCTION}

Short-range wireless technologies, such as Bluetooth [1] and IEEE 802.11b WLAN [4], are becoming pervasive in daily life applications, largely due to their very low deployment costs-such technologies use the unlicensed ISM frequency spectrum. However, using a unlicensed wireless resource is also a peril because coexistence interference is inevitable. Indeed, a number of previous studies have shown that this problem could be a severe one [2]. The mutual interference issues between Bluetooth and $802.11 \mathrm{~b}$ have drawn a lot of attention and it is so important that both parties, Bluetooth SIG (Special Interest Group) and IEEE 802.15 , have coordinated a task group to tackle this problem. The task group is known as Coexistence TG2 (Task Group 2) [5], which is responsible for quantifying the mutual interference issues between WLAN and WPAN

This research was supported by a grant from the Research Grants Council of the HKSAR under project number HKU 7162/03E.
The TG2's mission also includes developing a set of recommended practices and guidelines to facilitate coexistence between WLAN and WPAN. These kinds of suggested solutions are generally known as coexistence mechanisms [6].

Coexistence is defined by TG2 as the ability of one system to perform a task in a given (shared) environment where other systems may or may not be using the same set of rules. In fact, the concept of coexistence is not focused on mitigating the interference from a particular system's point of view but to make all systems nearby work peacefully. Thus, coexistence mechanisms are used to improve the performance of a system in the presence of interference as well as reducing the interference caused by itself on other devices actively operating in the ISM band. Usually, previously suggested coexistence mechanisms are to be implemented on the Bluetooth side in order to accommodate the existing or intervention of a WLAN system. They work at the MAC (Medium Access Control) layer to make adaptive control, tuning, and coordination, instead of making changes to the existing signal processing methods at the PHY (physical) layer. Coexistence mechanisms can be classified into two types according to their working principles: collaborative coexistence mechanisms and non-collaborative coexistence mechanisms.

\section{A. Collaborative Mechanisms}

Collaborative mechanisms require a communication pathway between $802.11 \mathrm{~b}$ and Bluetooth. With the traffic information of each party known beforehand, coexistence is accomplished by making the transmissions orthogonal in time domain. In fact, such a pre-condition generally requires both system modules to be colocated or within the same physical unit (e.g., within the same PC). Thus, colocated collaborative mechanisms are effectively a time domain MAC layer packet scheduling algorithm for managing the traffics of WPAN and WLAN. Indeed, a collaborative coexistence mechanism is particularly good for colocated application scenarios. Generally, it results in a better overall system performance. However, its applicability is limited because of the colocated constraint imposed. Under most practical circumstances, however, Bluetooth and $802.11 \mathrm{~b}$ are physically separated, and thus, it is nearly impossible for heterogeneous devices to communicate or exchange information. 


\section{B. Non-Collaborative Mechanisms}

Non-collaborative mechanisms work without any communication between $802.11 \mathrm{~b}$ and Bluetooth modules. As such, they achieve coexistence by carrying out two fundamental processes: channel classification and adaptive control actions.

Channel classification is the process used to estimate the channel conditions and to detect if there is any interference source nearby. Currently, all non-collaborative mechanisms share some general purpose and common methods for this process, like BER (Bit Error Rate), FER (Frame Error Rate), etc. Based on the results obtained in the channel classification process, the adaptive control actions will take the appropriate reactions accordingly. Different coexistence schemes employ very different adaptive control actions. For example, adaptive control actions for AFH (Adaptive Frequency Hopping) refers to changing the hopping sequence dynamically. On the other hand, in power control based coexistence mechanisms, adaptive control actions entail adjusting the transmission power according to the channel conditions.

Non-collaborative mechanisms are free from the constraints imposed on collaborative ones and such mechanisms are applicable to more application. scenarios. Its flexibility and practicality make it as the most important type of coexistence mechanisms. However, it cannot supersede collaborative ones since it is nearly impossible to be used in colocated scenarios. All in all, there is no single solution which can work for all possible scenarios. Because there are a variety of application scenarios, different types of coexistence mechanisms should be employed for different kinds of situations. In our study, we propose two non-collaborative mechanisms, which are detailed in Section III. The simulation results, generated using a detailed physical layer modeling with MatLab/Simulink [3], are provided in Section IV.

\section{Motivations of Our Research}

IEEE 802.15 TG2 has recommended AFH as the noncollaborative coexistence mechanism to harmonize the operation between WPAN and WLAN. The particular mechanism adopted by TG2 is called TG2 AFH.

However, in TG2 AFH, one of the major drawbacks is that the mechanism is only focused on the adaptive control actions process while the channel classification component is almost completely neglected in that the scheme just uses some general purpose measurement methods. Recall that channel classification and adaptive control actions are the fundamental steps in all non-collaborative coexistence mechanisms. Indeed, channel classification is particularly important since the appropriate adaptive reaction is taken based on the information provided by the channel classification process. Thus, there is a pressing need to design a fast and accurate channel classification method. However, the design in TG2 AFH would generally result in a slower response and less accurate channel classification results, which can adversely affect the system performance.
On the other hand, though some performance studies on TG2 AFH have been conducted by some other researchers, the performance analysis of $\mathrm{AFH}$ mechanisms under realistic resource constraints is by and large ignored by the research community. In the design of the TG2 AFH mechanism, it is observed that there is no explicit concern on the system resources required to run the coexistence mechanism. Indeed, despite the technology advancement and miniaturization of wireless portable devices, power and memory constraints are still always of prime concern. Thus, we strongly believe that resources consumption and implementation complexity should be taken into consideration when designing coexistence mechanisms for Bluetooth.

Furthermore, according to TG2, the objective of its AFH is used to achieve coexistence between WPANs and WLANs that operate in the same unlicensed band. However, TG2 AFH mechanism does not show any definite or direct consideration about WLAN in its design. In particular, it does not exploit the potential performance gain by taking into account the radio transmission characteristics of interference sources. In fact, we have found that such a practical consideration can reduce the time and space complexity of $\mathrm{AFH}$ mechanism.

Apart from the drawbacks in the existing approach, there are a number of open issues yet to be investigated. For example, the performance study on mutual interference between Bluetooth and $802.11 \mathrm{~b}$ is limited in scenarios. Previous studies were mainly focused on the relationship between interference levels at various distances. However, the effects of fluctuating interference level and spectrum congestion level in a small confined geographical environment are largely ignored.

On the other hand, TG2 has only recommended a frequency domain approach (TG2 AFH) coexistence mechanism but no other alternatives for choosing. There is no single omnipotent coexistence mechanism that can work for all possible scenarios. As there are a variety of potential application scenarios for Bluetooth and 802.11b operating simultaneously, we believe that a single solution is not enough and we should consider more different choices, e.g., time domain approaches.

Furthermore, to the best of our knowledge, there is no performance study comparing different types of coexistence mechanisms. We also cannot find any investigation about the best operating points for each type of coexistence mechanism, or when and which type of coexistence mechanisms should be used in terms of level of interference, separation, power level, data encapsulation methods, etc. Thus, a lot of work is needed in order to fill in these unexplored areas in coexistence study [2].

\section{Proposed Coexistence Approaches}

We propose two different coexistence mechanisms: ISOAFH (interference source oriented adaptive frequency hopping) and ISOMDMS (interference source oriented master delay MAC scheduling). 


\section{A. ISOAFH}

In our first approach, instead of using those standard and general purpose channel classification methods, we design a customized channel classification method for our ISOAFH mechanism. By using such a tailor-made channel classification method, the two mandatory steps for non-collaborative mechanisms-channel classification and adaptive control action - can be more tightly coupled, leading to higher efficiency yet lower implementation complexity.

In our customized channel classification method, we do not intend to find individual "bad" channels. Instead, we try to locate the carrier(s) of $802.11 \mathrm{~b}$ interference source(s) and then attempt to avoid hopping on all the affected Bluetooth channels. For instance, according to Table I, if we can be sure that the carrier of $802.11 \mathrm{~b}$ interference is $\mathrm{CH} 3$, then we can avoid hopping over Bluetooth $\operatorname{ch}(9-13)$ instead of taking measurements for individual channels. By doing this analysis, the device can respond to interference faster and obtain higher potential performance gain.

TABLE I

FrequenCy USAGE MAPPING.

\begin{tabular}{l|c|c}
\hline$G_{x}$ & 802.11 b freq. range $(\mathrm{MHz})$ & Bluetooth channels \\
\hline 1 & $2401-2423$ & ch $(0-21)$ \\
2 & $2406-2428$ & $\operatorname{ch}(4-26)$ \\
3 & $2411-2433$ & ch $(9-31)$ \\
4 & $2416-2438$ & ch $(14-36)$ \\
5 & $2421-2443$ & $\operatorname{ch}(19-41)$ \\
6 & $2426-2448$ & $\operatorname{ch}(24-46)$ \\
7 & $2431-2453$ & $\operatorname{ch}(29-51)$ \\
8 & $2436-2458$ & $\operatorname{ch}(34-56)$ \\
9 & $2441-2463$ & $\operatorname{ch}(39-61)$ \\
10 & $2446-2468$ & $\operatorname{ch}(44-66)$ \\
11 & $2451-2473$ & ch $(49-71)$ \\
\hline \hline
\end{tabular}

Specifically, our proposed channel classification method works as follows. We group Bluetooth channels into 11 groups according to the channel allocation of $802.11 \mathrm{~b}$, as shown in Table I. All channels affected by the same $802.11 \mathrm{~b}$ carrier are assigned to the same group. It should be noted that this group assignment is overlapping as well in that each Bluetooth channel can belong to more than one group (at most 5 groups). For example, Bluetooth ch10 belongs to Group 1, Group 2, and Group 3. Thus, instead of taking PER for each Bluetooth channel, we only keep track of the PER for that 11 groups only. This is formalized in the following rule.

1. For any channel within group $G_{x}$ resulted in error, the error rate is used for computing the aggregate PER for the whole $G_{x}$;

2. Besides, error in $\operatorname{ch}(i)$ is considered as the error for all groups containing $\operatorname{ch}(i)$;

3. If a group $G_{x}$ is found to have a PER of $\epsilon$, all channels in group $G_{x}$ are labeled as "bad" channels.

For example, if a packet sent in Bluetooth channel $\operatorname{ch}(38)$ gets corrupted, this packet error is counted in the PER of Groups 5, 6, 7, and 8. For another example, if Bluetooth channel $\operatorname{ch}(32)$ gets interfered, then the packet error is contributed to PER reading of Group 4, 5, 6, and 7. By doing this, the group corresponding to the carrier of the interference source will have the highest packet error rate value. Thus, the Bluetooth system can locate the carrier of the interference and try to avoid hopping in the whole range of engaged frequencies.

Moreover, compared to the channel-by-channel PER measurement in TG2 AFH, this mechanism can respond faster to interference since the statistics build up quickly. This is because one error can be counted in more than one group and all errors within a group contribute to a single PER reading.

To implement the measurement of PER, it can utilize the ARQ (Automatic Repeat reQuest) scheme in ACL link. This scheme involves an acknowledgment bit (ARQN) to let the master know whether the last packet was received correctly. The recipient checks each received packet for error, and if error is detected, it indicates this in the header of the return packet. Afterwards, the sender retransmits the packet. Thus, by making use of this scheme, the PER can be measured readily at the sender side.

\section{B. ISOMDMS}

There are many practical difficulties in the implementation of $\mathrm{AFH}$ in a real life environment [2]. Among all the factors, the most important issues are the requirement of hardware modifications and backward incompatibility. Hardware modifications, that are added to support AFH, inevitably lead to a higher cost. Another implication of such change is that AFH cannot be used unless both master and slave are AFH enabled. However, there are many legacy Bluetooth devices in use and these devices cannot make use of $\mathrm{AFH}$.

Due to these practical limitations imposed on AFH, we consider another non-collaborative coexistence mechanism that possesses properties of both ease of implementation and backward compatibility with legacy Bluetooth devices. In view of these motivations, a time domain noncollaborative coexistence approach, called Master Delay MAC Scheduling (MDMS), is also proposed in this paper in order to satisfy the concerns mentioned above.

Figure 1 shows the basic functionality of MDMS. For illustration purposes, let us assume that the Bluetooth system is fully loaded (i.e., keep on transmitting) using a single-slot packet type. As can be seen in Figure 1, the sequence of numbers represents the hopping frequencies to be used for every individual packet in each time slot period. First, based on some channel classification technologies, the device (i.e., the Bluetooth master) obtains the channel conditions and hence, realizes which channels are being engaged by other nearby systems. Second, the master checks against the hopping sequence in pairs (i.e., to protect the " $\mathrm{Tx}$ " and "Rx" transmission pattern over ACL links) with the channel state information (CSI). Once any of the assigned channels is found to be in the set of "used" 
(i.e., "bad") channels, no transmission is allowed in the corresponding time slot, and more importantly, the affected packet is delayed until the next time slot with a possibly "unused" (i.e., "good") channel.

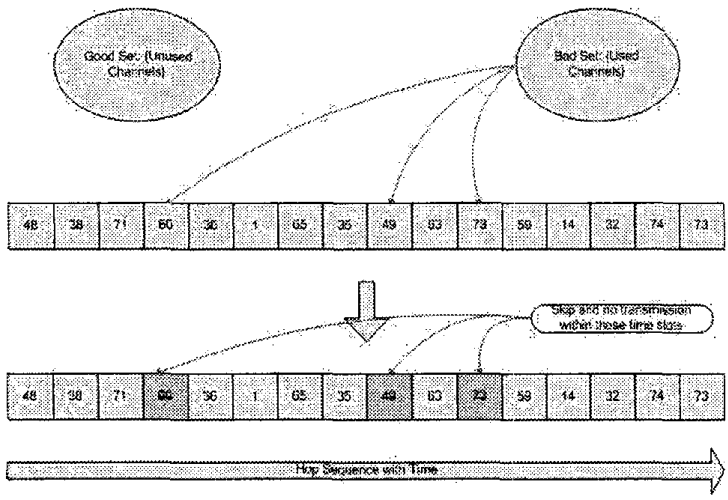

Fig. 1. An illustration of the principle of MDMS.

As described above, the working principle of MDMS is quite simple. MDMS does not impose any change to the original pseudorandom hopping sequence like AFH. However, it can still fulfill the coexistence goals by deferring the transmission if the assigned frequencies are sensed to be occupied. In this manner, Bluetooth devices schedule its traffic in a non-collaborative manner and effectively reduce the offered load during possible collision periods. Thus, compared to AFH, MDMS is a conservative approach in the sense that AFH always aggressively tries to search for a "good" channel while MDMS just tries to stop transmission on interfered channels.

On the other hand, since a Bluetooth master has complete control over the piconet's traffic and the MDMS scheme does not change the original pseudorandom hopping sequence, only the Bluetooth master needs to be MDMS enabled. Yet the whole piconet can enjoy the coexistence performance gain. This meets the requirement on backward compatibility. Furthermore, the adaptive control actions of MDMS are some simple traffic control actions on the time domain and as such, they can be easily implemented by slightly upgrading existing Bluetooth MAC layer firmware. Thus, MDMS also fulfills the condition of ease of implementation.

\section{Simulation Results}

Figure 2 compares the collision rate of all the frequency hopping mechanisms in a heterogeneous environment where multiple $802.11 \mathrm{~b}$ and Bluetooth connections coexist.

It can be seen that the collision rate of all AFH mechanisms increases with the number of piconets and the collision rate is larger than the total of individual environments (i.e., collision rate of heterogeneous environment is greater than collision rate of inter-piconets only plus collision rate of the case with $802.11 \mathrm{~b}$ networks only). This is due to the fact that interference from other piconets adversely affects the channel classification process of AFH mechanisms, and makes them fail to identify potential wideband and nonstatic interference sources and hence, results in a higher overall collision rate.

Figure 3 shows the throughput of Bluetooth system generated by different types of non-collaborative coexistence mechanisms under different activity level of interference (1-100\%) and different congestion levels in the ISM band (with one to three 802.11b connections) for DH1, DH3, and DH5 packet types, respectively. For the corresponding mean access delay results, the reader is referred to [2].

In Figure 3(a) single slot packets are used. When $802.11 \mathrm{~b}$ interference is low (e.g., $<30 \%$ ), both ISOMDMS and TG2 $\mathrm{AFH}$ have more or less the same throughput since the effect of the interference is limited at such a low level of activity, independent of the channel estimation algorithm used. When the interference level increases, ISOMDMS responds in such a way that it realizes the "bad" channels/interference faster, and hence, starts to skip the transmission period with bad frequencies assigned. This behavior results in a reduced level of throughput. For TG2 AFH, although it is expected to respond slower than ISOMDMS, the throughput of TG2 AFH is still slightly higher than ISOMDMS as TG2 AFH always hops to a "good" channel.

When the interference level keeps on increasing to a high level (e.g., > 60\%), both mechanisms can respond to the interference in a shorter time and take the corresponding adaptive actions. However, TG2 AFH always attempts to find a "good" channel while ISOMDMS stops its transmission, and thus, the difference between their throughputs further increases.

In Figures 3(b) and (c), multi-slot packets are used. It can be observed that, compared to DH1, the throughput difference in each simulation step is relatively larger, since each packet loss corresponds to a larger amount of data loss. When the interference level is lower than $60 \%$, surprisingly, we can see that the throughput of ISOMDMS is higher than that of TG2 AFH. It can be explained as follows. At a low level of interference activity, TG2 AFH needs to take a longer time to detect the presence of interference. Thus, TG2 AFH results in a higher packet loss before taking any adaptive control actions. However, ISOMDMS can response to interference faster and take the appropriate traffic control actions.

\section{REFERENCES}

[1] Bluetooth Special Interest Group, Specifications of the Bluetooth Systems, vol. 1, v.1.0B Core, Dec. 1999.

2] M. C.-H. Chek, Coexistence Techniques for Heterogeneous Mobile Devices Operating in Uncoordinated Radio Spectrum, M.Phil. Thesis, The University of Hong Kong, Dec. 2003.

[3] Developers of MATLAB and Simulink for Technical Computing, http://www. mathworks. com/index.shtml, 2003.

[4] IEEE Std. 802.11b, "Part 11: Wireless LAN Medium Access Control (MAC) and Physical Layer (PHY) Specifications: Higher-Speed Physical Layer Extension in the 2.4GHz Band," Sept. 1999.

[5] IEEE 802.15 WPAN Task Group 2 (TG2) http://ieee802.org/15/pub/TG2.html, 2003.

[6] IEEE 802.15.2 Coexistence Mechanisms, http://www . ieee802.org/15/pub/TG2-Coexistence-Mechanisms.ht: 2003. 


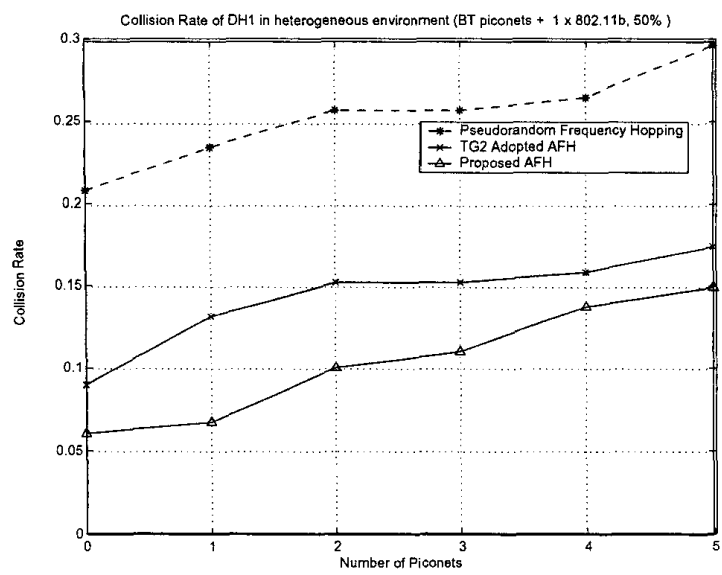

(a) $\mathrm{DH} 1,50 \% 802.11 \mathrm{~b} \times 1$

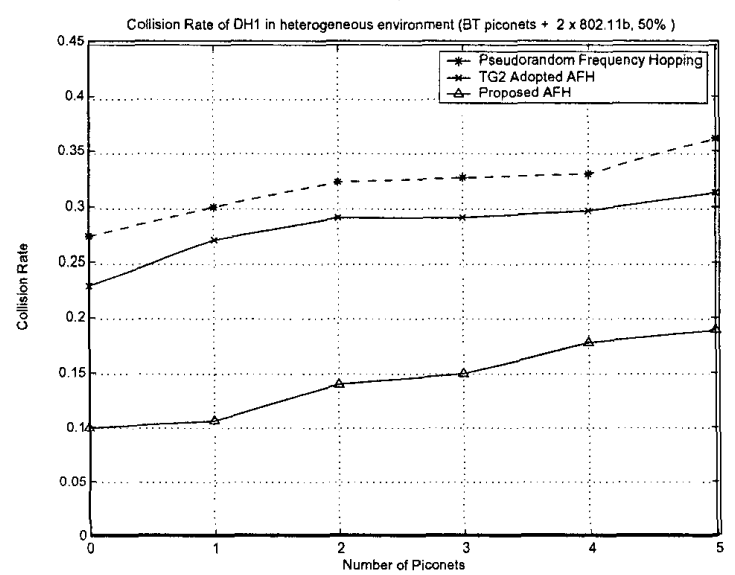

(b) $\mathrm{DH} 1,50 \% 802.11 \mathrm{~b} \times 2$

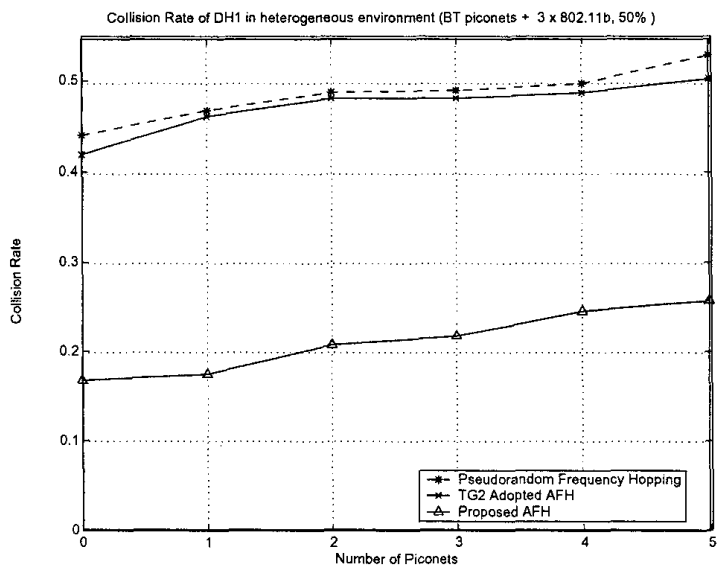

(c) DH1, $50 \% 802.11 \mathrm{~b} \times 3$

Fig. 2. Comparison of the collision rate of all the frequency hopping mechanisms in the heterogeneous interference environment.

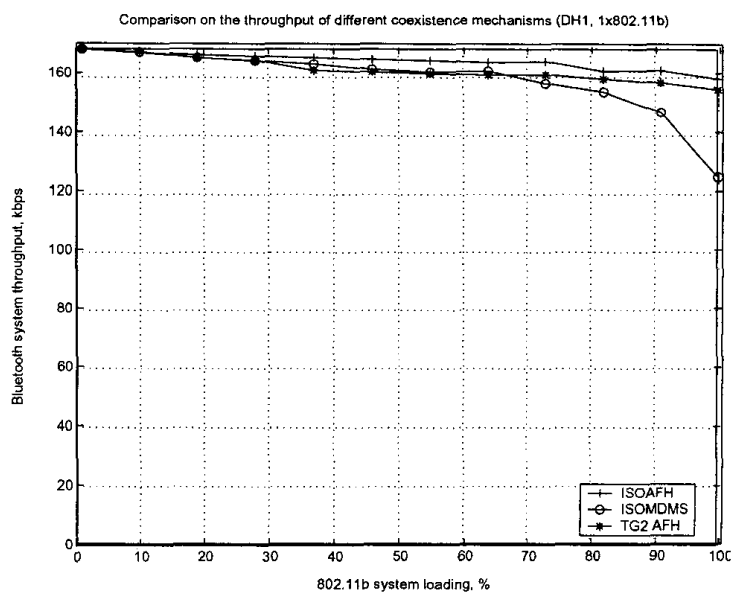

(a) $\mathrm{DH} 1$

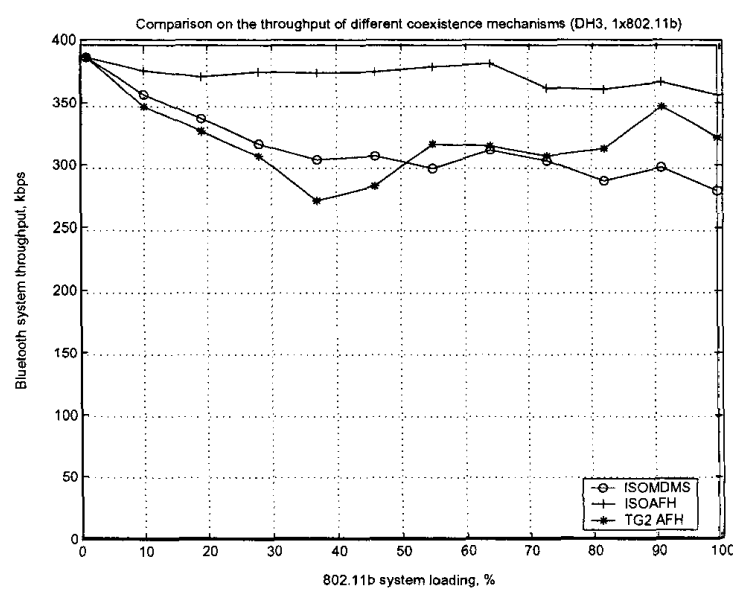

(b) $\mathrm{DH} 3$

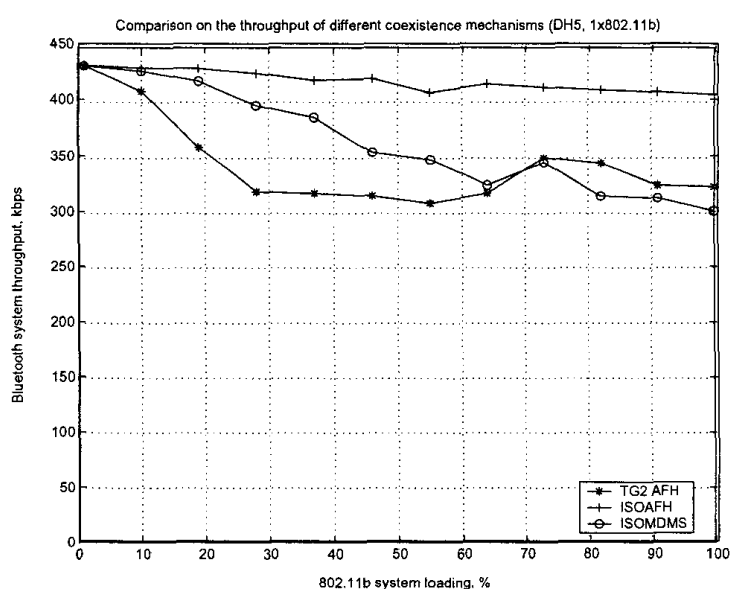

(c) DH5

Fig. 3. Comparison of throughput using different types of noncollaborative coexistence mechanisms. 